\title{
IMPLANTE DE CONDUCTO APICOAÓRTICO EN PACIENTE CON ESTENOSIS AÓRTICA SEVERA POST REEMPLAZO VALVULAR PROTÉSICO*
}

\author{
Drs. Juan Salman A. ${ }^{1}$, Juan Carlos Bahamondes S. ${ }^{1}$, \\ Abelardo Silva V. ${ }^{1}$, Joaquín Gundelach M. ${ }^{1}$, Andrés Díaz H. ${ }^{1}$
}

1 Servicio de Cirugía Cardiovascular, Centro Cardiovascular Hospital Regional. Departamento de Cirugía. Facultad de Medicina. Universidad de La Frontera.

Temuco, Chile.

\section{Abstract \\ Apicoaortic valved conduit implanted in a patient with severe aortic stenosis due to mismatched aortic valve prosthesis}

Introduction: The most frequent surgical procedure of severe left ventricular outflow tract obstruction due to severe aortic stenosis is aortic valve replacement, but there is an alternative and less conventional method described by Cooley used in patients with previous aortic valve replacement and severe aortic wall calcification that bypasses the left ventricular outflow tract through the implant of an apicoaortic valved conduit. Clinical case: We describe the case of 42 years old patient with severe aortic stenosis due to mismatched valve prosthesis previously installed treated by of apicoaortic conduit implanted through left posterolateral thoracotomy.

Key words: Aortic valve stenoses, (MeSH), heart valve prosthesis implantation (MeSH), left ventricular outflow obstruction (MeSH).

\section{Resumen}

Introducción: La estenosis aórtica fibrocálcica es el tipo de valvulopatía más frecuente, especialmente en los adultos mayores, siendo el reemplazo valvular aórtico el tratamiento definitivo. Un mal resultado post operatorio puede deberse a un mal funcionamiento de la válvula protésica. Caso clínico: Paciente de 42 años de edad sometido a reemplazo valvular aórtico por presentar estenosis aórtica severa fibrocálcica en una válvula bicúspide y aorta de pared muy fina. Evoluciona asintomático durante 4 años, al cabo de los cuales y luego de abandonar su tratamiento anticoagulante consulta por disnea y dolor anginoso. Un ecocardiograma muestra estenosis severa de la válvula protésica. Dado los antecedentes de su cirugía previa se decide

*Recibido el 14 de mayo de 2013 y aceptado para publicación el 17 de junio de 2013.

Este estudio no tuvo apoyo financiero. Los autores no declaran conflictos de interés.

Correspondencia:

Dr. Juan Salman A.

Av. Manuel Montt $112.4^{\circ}$ piso, Temuco, Chile. Fax: 5645325760

juansalman@gmail.com 
implantar un conducto de dacrón valvulado ápico-aórtico a través de toracotomía posterolateral izquierda. Evolución post operatoria muy satisfactoria permaneciendo asintomático, con un seguimiento de 15 meses. La recidiva de la estenosis aórtica severa post reemplazo valvular protésico tiene en el bypass ápico-aórtico una excelente alternativa de tratamiento efectivo, especialmente cuando el recambio valvular tradicional implica un alto riesgo quirúrgico.

Palabras clave: Estenosis aórtica, cirugía, bypass ápico-aórtico.

\section{Introducción}

La estenosis aórtica se ha convertido en el tipo más frecuente de valvulopatía, especialmente en adultos sobre los 65 años, presentándose con frecuencia como estenosis aórtica severa fibrocálcica. El reemplazo valvular aórtico es el tratamiento definitivo.

Un mal resultado postoperatorio puede deberse a complicaciones relacionadas con un mal comportamiento del funcionamiento de la válvula protésica y evidente compromiso hemodinámico del paciente. Esto último ha llevado a la utilización de nuevas técnicas quirúrgicas para el tratamiento de la recidiva de la estenosis aórtica severa, siendo una alternativa descrita el implante de un conducto valvulado ápicoaórtico, especialmente, cuando el recambio valvular tradicional implica un alto riesgo quirúrgico.

El objetivo de este trabajo es comunicar los resultados en un paciente con estenosis aórtica severa post reemplazo valvular aórtico tratado mediante esta técnica.

\section{Caso clínico}

Varón de 42 años portador de enfermedad valvular aórtica con estenosis severa, que en diciembre de 2005 se realiza reemplazo valvular aórtico con válvula mecánica St. Jude Nº17, en el Hospital Dr. Hernán Henríquez Aravena, destacando durante la cirugía una válvula aórtica bícúspide, anillo pequeño muy rígido e intensamente calcificado al igual que los velos aórticos. La aorta se apreció de paredes muy finas y estructuralmente de mala calidad. Se practicó sutura de pared aórtica con puntos corridos de prolene 4/0 en dos planos, sobre bandas de teflón y abundante impregnación de hemostático biológico bioglue.

En octubre de 2009 consulta por disnea progresiva y dolor anginoso de dos meses de evolución; había abandonado desde hacía dos meses el tratamiento anticoagulante. Un ecocardiograma transtorácico evidencia prótesis valvular aórtica disfuncionante por estenosis severa (gradiente máximo $107 \mathrm{mmHg}$, gradiente medio $60 \mathrm{mmHg}$ ) sin insuficiencia.

Se optimiza el tratamiento médico citándose a nuevo control con ecocardiograma en enero de
2010 que informa un gradiente aórtico máximo de $115 \mathrm{mmHg}$ y medio de $81 \mathrm{mmHg}$ con una fracción de eyección del ventrículo izquierdo (VI) de $61 \%$. Se efectúa coronariografia informada como normal y se opta nuevamente por manejo quirúrgico. Se discute el caso y dado los antecedentes de su cirugía previa y el riesgo altamente prohibitivo de realizar una resustitución protésica tradicional se planteó al paciente implantar un conducto de dacrón valvulado ápico-aórtico. El paciente firmó el consentimiento informado y la operación se practicó en agosto de 2010 .

\section{Técnica quirúrgica}

Toracotomía posterolateral izquierda por el quinto espacio intercostal accediéndose a aorta descendente y ápex ventricular izquierdo abriendo el pericardio. Conjuntamente se exponen los vasos femorales izquierdos para canulación y conexión a circulación extracorpórea (CEC). Iniciada ésta se practica apertura del ventrículo izquierdo a 2 centímetros lateralmente del ápex donde se implanta una prótesis de dacrón de $24 \mathrm{~mm}$ mediante puntos separados de prolene 3/0 apoyados sobre pledgets. Clampeo parcial de aorta descendente distal, procediéndose a anastomosar termino-lateral un conducto valvulado St Jude $n^{\circ} 21$ con prolene 3/0 sutura continua, Retiro del clamp parcial. Anastomosis de ambos conductos con prolene $3 / 0$, para luego restablecer la función cardíaca normal. Retiro de CEC y de cánulas femorales. Cierre del tórax en forma habitual.

Evolución post operatoria inmediata sin incidentes, arritmias ni otros eventos cardiovasculares. Un ecocardiograma transtorácico a la semana post cirugía, evidencia prótesis aórtica con gradiente máximo de $44,9 \mathrm{mmHg}$ y medio de $24,8 \mathrm{mmHg}$. Angio TC de tórax muestra conducto ápico-aórtico valvulado in situ sin complicaciones y sin presencia de fugas periprotésicas (Figuras 1 y 2 ) siendo dado de alta al octavo día del post-operatorio.

\section{Seguimiento}

Se hizo mediante controles clínicos periódicos en policlínico de cirugía cardíaca y/o de las consultas de médicos tratantes durante 15 meses, manteniéndose asintomático, en capacidad funcional I de la NYHA. 


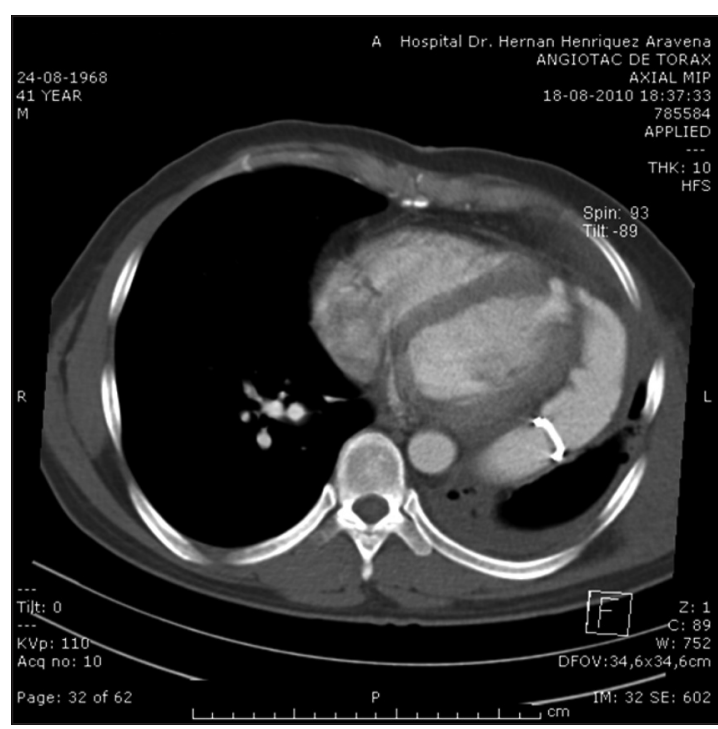

Figura 1. Angio TC de tórax de control post operatorio.

\section{Discusión}

La idea de que puede crearse otro tracto de salida del ventrículo izquierdo con la finalidad de aliviar una obstrucción compleja intraventricular no es nueva. En 1910 Alexis Carrel describió su trabajo experimental con un puente entre el VI y la aorta torácica ${ }^{1}$. En 1955 Sarnoff y cols, fueron capaces de demostrar, utilizando una válvula de Hufnagel incorporada en un tubo de lucita, que se podía derivar todo el flujo sanguíneo desde el VI hacia la aorta torácica ${ }^{2,3}$. En 1955, Templeton implantó el conducto diseñado por Sarnoff en 5 pacientes sobreviviendo uno de ellos más de diez años, experiencia que nunca fue publicada ${ }^{4-6}$.

En 1975, Bernhard utilizó un injerto de dacrón valvulado que implantó entre el vértice del VI y la aorta descendente en un paciente de 22 años de edad que sufría de hipoplasia del anillo de la válvula aórtica y de la aorta ascendente logrando con éxito aliviar el tracto de salida del $\mathrm{VI}^{5}$. Ese mismo año, Cooley implantó un tubo valvulado de dacrón entre en el vértice del VI y la aorta abdominal en una paciente de 10 años de edad que padecía una estenosis aórtica severa y a la cual se le había practicado, 4 años antes, una valvulotomía aórtica ${ }^{6}$. En 1976, Dembitsky y Weldon, implantaron este mismo conducto en 2 pacientes con estenosis subaórticas hipertróficas irresecables, llevando el extremo distal del conducto a la aorta abdominal infrarrenal ${ }^{7}$. Renzulli y cols, entre diciembre de 1977 y julio de 1983, recopilaron 4 pacientes intervenidos por estenosis

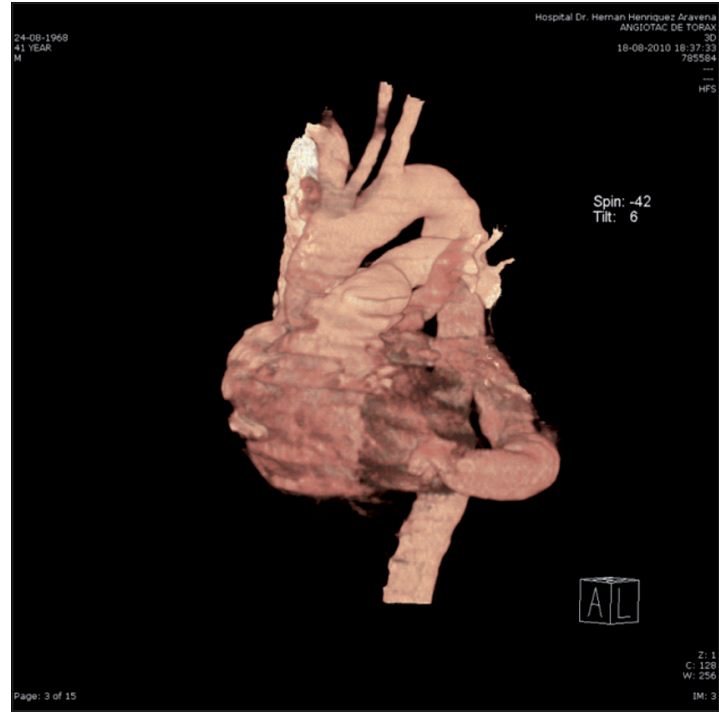

Figura 2. Reconstrucción tridimensional post implante de conducto ápico-aórtico.

subaórtica hipertrófica idiopática y cuyas características anatómicas eran inapropiadas para la operación de Morrow, por lo cual implantaron el conducto ápico-aórtico anastomosando el extremo distal en la aorta ascendente en 3 casos y en la aorta abdominal superior en el cuarto. En dos de estos pacientes, se constató una sobrevida de 15 y 19 años respectivamente $^{8,9}$. En 1980, Normand, Nihill y Cooley presentaron en un artículo de revisión, 27 pacientes que padecían de estenosis aórtica congénita y obstrucciones múltiples complejas del tracto de salida del VI tanto en pacientes pediátricos como en adultos en los cuales la anastomosis distal del conducto de dacrón valvulado se practicó en la región supracelíaca de la aorta abdominal. La mortalidad perioperatoria fue $11 \%$ y la mortalidad tardía similar ${ }^{10}$.

Entre los años 2003 y 2007, Gammie y su grupo recopilaron los resultados del implante del conducto ápico-aórtico en 31 pacientes con estenosis aórtica severa y alto riesgo para el reemplazo valvular aórtico. La edad promedio fue 81 años. La mortalidad perioperatoria fue de un $13 \%$, aunque en los últimos 16 pacientes, no hubo mortalidad. En todos los casos y a diferencia de las experiencias previas, el abordaje se hizo a través de una toracotomía posterolateral izquierda y la anastomosis distal se realizó en la aorta descendente ${ }^{11}$.

El abordaje transtorácico es muy bien tolerado por los pacientes, ofrece un acceso directo a la aorta torácica descendente y se evita una nueva esternotomía. Del mismo modo, la eficacia y durabilidad del conducto ápico-aórtico valvulado sobre los síntomas 
fue confirmada con una mejoría significativa en la función postoperatoria y calidad de vida de los pacientes. Además el seguimiento ecocardiográfico demostró un gradiente bajo entre el VI y la aorta con diámetros ventriculares prácticamente normales. Ninguno de estos pacientes experimentó arritmia ventricular maligna.

El conducto ápico-aórtico ha sido utilizado con éxito, en alteraciones congénitas de la válvula aórtica y tracto de salida del VI, valvulopatía aórtica adquirida, cirugía previa de la válvula aórtica, patología de la aorta ascendente como aorta de porcelana y en casos de pericarditis. Así mismo se puede utilizar en enfermos con una discordancia severa entre prótesis y paciente (situación en la que tras un remplazo valvular aórtico, el área valvular funcional es menor que la de una válvula humana normal; Rahimtoola 1978), evitando la resustitución valvular convencional con ampliación del anillo aórtico ${ }^{12}$.

De acuerdo a estas consideraciones, buena tolerancia y excelente evolución post operatoria, no es aventurado manifestar, que el implante del conducto apico-aórtico por vía transtorácica se convierta en una alternativa quirúrgica, que a futuro, probablemente competirá con el reemplazo valvular aórtico percutáneo (RVAP) ya que tiene claras ventajas sobre éste. Permite usar varios tipos y tamaños de prótesis, ha demostrado su eficacia y durabilidad, tiene menos riesgos de ictus peri procedimientos, no entraña riesgo de bloqueo aurículo ventricular completo (AVC) como tampoco presenta leak periprotésico. Estos efectos adversos fueron remarcados recientemente en una revisión que incluyó 49 estudios con más de 16.000 pacientes sometidos a RVAP y donde se muestra el promedio de las complicaciones más frecuentes del RVAP: bloqueo AVC $(13,1 \%)$, complicaciones vasculares $(10,4 \%)$, insuficiencia renal aguda que requirió diálisis $(4,9 \%)^{13}$.

Por otro lado el conducto apico-aótico se realiza con corazón latiendo evitando la isquemia miocárdica y la función del VI no se afecta por la inserción en el ápex de este conducto. De igual manera, pensamos que hasta que el RVAP se haga rutinario, el conducto ápico-aórtico por vía transtorácica podría ser considerado para tratar a pacientes con estenosis aórtica severa y alto riesgo quirúrgico (edad avanzada y comorbilidad como falla renal, patología de la aorta ascendente, etc.) que se rechazan para cirugía de reemplazo.

Este caso que presenta nuestro grupo corresponde al primero documentado a nivel nacional con esta técnica, con excelente resultado, evitando una resustitución valvular protésica tradicional que en nuestro paciente habría conllevado un alto riesgo quirúrgico y muy dudosas posibilidades de éxito.

\section{Referencias}

1. Carrel A. Experimental surgery of the aorta and heart. Ann Surg. 1910;52:83-95.

2. Sarnoff SJ, Donovan TJ, Case RB, Waithe PE. Technical Assistance: The surgical relief of aortic stenosis by means of apical aortic valvular anastomosis. Circulation 1955;11:564.

3. Hufnagel CA. Aortic plastic valvular prosthesis. Preliminary report. Bulletin of the Georgetown University Medical Center 1951;4:128.

4. Templeton JY. Personal Communication to Drs. Cooley and Norman, 1975 (cita textual que aparece en la bibliografía $\mathrm{N}^{\circ} 6$ de estas referencias).

5. Bernhard WF, Poirier V, La Farge GC. Relief of Congenital Obstruction to left Ventricular Outflow with a Ventricular-Aortic Prosthesis. J Thorac Cardiovasc Surg. 1975;69:223-9.

6. Cooley DA, Norman JC, Mullins CE, Grace RR. Left ventricle to abdominal aorta conduit for relief of aortic stenosis. Bull Texas Heart Inst. 1975;2:376-83.

7. Dembitsky WP, Weldon CS. Clinical experience with the use of a valve-bearing conduit to construct a second left ventricular outflow tract in cases of unresectable intraventricular obstruction. Ann Surg. 1976;184:31723.

8. Morrow AG. Hypertrophic subaortic stenosis. Operative methods utilized to relieve left ventricular outflow obstruction. J Thorac Cardiovasc Surg. 1978;76:423-30.

9. Renzulli A, Rosario Gregorio R, De Feo M, Ismeno G, Covino FE, Cotrufo M. Long-term results of apicalaortic valved conduit for severe idiopathic hypertrophic subaortic stenosis. Texas Heart Institute Journal 2000; 27:24-8.

10. Norman JC, Nihill MR, Cooley DA. Valved apico-aortic composite conduits for left ventricular outflow tract obstructions. A 4 year experience with 27 patients. Am J Cardiol. 1980;45:1265-71.

11. Gammie JS, Krowsoski LS, Brown JM, Odonkor PN, Young CA, Santos MJ, et al. Aortic valve bypass surgery. Midterm clinical outcomes in a high-risk aortic stenosis population. Circulation 2008;118:1460-6.

12. Rahimtoola SH. The problem of valve prosthesis-patient mismatch. Circulation 1978;58;20-4.

13. Khatri PJ, Webb JG, Rodés-Cabau J, Fremes SE, Ruel M, Lau K, et al. Adverse effects associated with transcatheter aortic valve implantation: a meta-analysis of contemporary studies. Ann Intern Med. 2013;158:3546. 